Review Article

\title{
Resting-State fMRI in Studies of Acupuncture
}

\author{
Xiaoling Li, ${ }^{1}$ Lina Cai, ${ }^{2}$ Xiaoxu Jiang, ${ }^{2}$ Xiaohui Liu, ${ }^{2}$ Jingxian Wang, ${ }^{2}$ Tiansong Yang $\mathbb{D},{ }^{1}$ \\ and Feng Wang $\mathbb{B}^{1}$ \\ ${ }^{1}$ First Affiliated Hospital, Heilongjiang University of Chinese Medicine, Harbin, China \\ ${ }^{2}$ Heilongjiang University of Chinese Medicine, Harbin, China \\ Correspondence should be addressed to Tiansong Yang; yangtiansong2006@163.com and Feng Wang; wfzmy123@163.com
}

Received 20 December 2020; Revised 10 February 2021; Accepted 28 February 2021; Published 23 March 2021

Academic Editor: Wen Si

Copyright (c) 2021 Xiaoling Li et al. This is an open access article distributed under the Creative Commons Attribution License, which permits unrestricted use, distribution, and reproduction in any medium, provided the original work is properly cited.

Research exploring the mechanism of acupuncture has been a hot topic in medicine. Resting-state functional magnetic resonance imaging (rs-fMRI) research is a noninvasive and extensive method, which is aimed at the research of the mechanism of acupuncture. Researchers use fMRI technologies to inspect the acupuncture process. The authors reviewed the application of rsfMRI in acupuncture research in recent 10 years from the aspects of studying acupoints, subjects, acupuncture methods, and intensities. The results found that the application of rs-fMRI in acupuncture research mainly includes research on the onset mechanism of acupuncture treatment; visual evidence of diagnosis and treatment of dominant diseases; efficacy assessments; physiological mechanism of acupoint stimulation; and specific visualization of acupoints.

\section{Introduction}

Acupuncture has been used as a traditional treatment method and is now becoming popular rapidly in clinical medicine practice because of its undeniable therapeutic effects $[1,2]$. However, the effect of the acupuncture mechanism on the central system is still unclear, so the exploration of the acupuncture efficacy mechanism has been a hot topic in the medical study $[3,4]$. Nowadays, restingstate $\mathrm{fMRI}$ is a prominent technique to measure the activities in the brain caused by acupuncture $[5,6]$.

The objective of this study is to develop an artificial intelligence analysis algorithm and automatic result output system with the function of reading and analyzing fMRI images in acupuncture research. Based on this demand, we preliminarily collected the contents mentioned in this review.

\section{Comparison of Acupoints}

2.1. Comparison of a Single Acupuncture Acupoint vs. Acupoint or Nonacupoint. Eight recent studies showed that acupuncture at different acupoints could induce different activities of brain regions in resting-state networks (Table 1). Zhong et al. [7] found that the connectivity between the superior temporal gyrus and anterior insula was enhanced by acupuncture at GB40 and the connection between the STG and postcentral gyrus was increased following acupuncture at KI3. Qiu et al. [8] reported the differences in wavelet transform coherence characteristic curves in the declive, precuneus, postcentral gyrus, supramarginal gyrus, and occipital lobe with acupuncture at LR3 and nonacupoint, and the posteffect can last for 5 minutes. Feng et al. [9] found that compared to nonacupoint, the increase in correlations for acupoints was related with the limbic/paralimbic and subcortical regions and the decrease in correlations was related with the sensory and frontal cortex. Long et al. [10] found that the areas with significant changes in functional connection values after acupuncture at ST36 were mainly concentrated in the middle temporal gyrus and parahippocampal gyrus, which are the main hubs of the default network. The therapeutic effect of acupuncture on pain may be related to the enhanced connection between these two areas. Yu et al. [11] observed that acupuncture at LR3 mainly activated the brain functional network in visual function, associative function, and emotional cognition, which was consistent with the therapeutic effect of LR3 in traditional medicine; furthermore, it had specific values to interpret the acupoint specificity of the LR3. Cai et al. [12] 
TABLE 1: Research studies on a single acupuncture acupoint vs. acupoint or nonacupoint.

\begin{tabular}{|c|c|}
\hline Acupoints & Main findings \\
\hline GB40 vs. KI3 [7] & Superior temporal gyrus (STG), auditory network, and anterior insula vs STG and postcentral gyrus \\
\hline LR3 vs. nonacupoint [8] & $\begin{array}{c}\text { Declive, precuneus, postcentral gyrus, supramarginal gyrus, and occipital lobe; post-ACU can lasted for } \\
5 \text { minutes }\end{array}$ \\
\hline ST36 vs. nonacupoint [9] & Limbic/paralimbic regions \\
\hline ST36 vs. nonacupoint [10] & Parahippocampal gyrus and middle temporal- major hubs of the default mode network \\
\hline LR3 vs. nonacupoint [11] & Network: visual, emotion, and cognition \\
\hline $\begin{array}{l}\text { Back-shu + front-mu vs. } \\
\text { single [12] }\end{array}$ & ReHo: thalamus, the posterior cingulate gyrus, and the precuneus \\
\hline ST36 vs. nonacupoint [13] & Somatosensory and saliency processing regions \\
\hline Combinations vs. single [14] & More widely activate areas; new brain areas; and curative effects \\
\hline
\end{tabular}

found that needling at the back-shu and front-mu points of the stomach can induce the ReHo changes in the thalamus, the posterior cingulate gyrus, and the precuneus brain regions compared with the single point, and these areas are the important brain regions for points to regulate gastric motility. Nierhaus et al. [13] considered that the stimulation about ST36 can obviously modulate somatosensory and saliency processing activities than nonacupoint. Liu et al. [14] addressed that the combined acupoints activate a wider range of brain areas than single points. In addition, the association of acupoints could activate a few new brain areas and engender new curative effects.

2.2. Comparison of before Acupuncture vs. after Acupuncture. More and more results suggested the brain activity after acupuncture was different from that before acupuncture, needing at points could change inherent activities of the cerebral cortex (Table 2). Zhang et al. [15] revealed that needling at LR3 and KI3 specifically promoted the function of brain areas, which are related to vision, emotion, and cognition, and inhibited associated with attention, phonological, and semantic processing. Zhou et al. [16] found that acupuncture at the points of the Lung Meridian could significantly strengthen ALFF in the right gyrus subcallosum and inferior frontal gyrus and weaken in the right postcentral gyrus, left precuneus, superior temporal gyrus, and middle temporal gyrus and needling at the Lung Meridian points could alter inherent activities of the cerebral cortex. Liu et al. [17] observed that compared with before acupuncture, the ReHo in cognitive network, motor network, default network, and limbic system encephalic changed after acupuncture at GB34.

2.3. Comparison of Verum Acupuncture vs. Sham Acupuncture. Table 3 presents that verum acupuncture was superior to sham acupuncture. Verum acupuncture stimulated acupoints to produce the sensation of Deqi. Zyloney et al. [18] reported that verum acupuncture (ACU) generated the connectivity in the PAG, PCC, and precuneus, contrasting with sham ACU, and significantly promoted the changes of functional connectivity regions in the pain matrix and default mode network by acupuncture at LR3. Zhao et al. [19] indicated that a true acupuncture effect had more extensive and more significant brain reactions in the long- term stimulation than sham ACU. Adopting the ALFF and ReHo approach, Wu et al. [20] observed that acupuncture at LR3 can modulate the activities of functional brain regions, such as vision, movement, sensation, emotion, and analgesia, but sham ACU does not show correlations in regions with functions. Liu et al. [21] found that poststimulus effects showed a more significant characteristic through verum ACU, and the insula plays a crucial role in the switch process of immediate- and delayed-effect neural responses of acupuncture.

\subsection{Comparison of Contra-Acupuncture vs. Ipsiacupuncture.}

The authors used contra-acupuncture vs. ipsiacupuncture to measure the mechanism of acupoints (Table 4). Using ReHo as the observation index, Zhang et al. [22] found that ACC plays an essential role in the regulation of contralateral ST38, on the other pathway of the brainstem-thalamus-cortex, which is an important region on the ipsilateral ST38. Yan et al. [23] revealed the different changes of brain functional connectivity modes after acupuncture at contralateral or ipsilateral ST38, which supported the hypothesis of acupoint specificity.

Two studies designed the comparison of individual differences. Yang et al. [24] found the decreased connectivity in the left middle frontal gyrus in Val/Val homozygous subjects compared with the Val/Val homozygous subjects. The change of ReHo in different brain regions may be related to different constitution groups, and the acupuncture feeling of physical differences may be an important factor affecting acupuncture analgesia [25].

\section{Comparison of Different Acupuncture Methods}

Ten studies to investigate the verity of brain activities used different acupuncture methods (Table 5). The DMN was observed to have a more extensive connectivity following MA and EA, and the connectivity in the sensorimotor network was specifically increased by TEAS [26]. Lv et al. [27] found the changes in the ReHo or ALFF value in brain region functions related to cognitive after-laser acupuncture at TGA. Jiang et al. [28] reported more prominent connectivity between the DMN and the SMN during 30 minutes transcutaneous electrical nerve stimulation (TEAS) compared with MTEAS. Motor function regions 
TABLE 2: Research studies on before acupuncture vs. after acupuncture.

\begin{tabular}{|c|c|}
\hline Study & Main findings \\
\hline Zhang et al. [15] & $\begin{array}{l}\text { ALFF: increased cerebral occipital lobe and middle occipital gyrus } \\
\text { Decreased gyrus rectus of the frontal lobe and posterior lobe }\end{array}$ \\
\hline Zhou et al. [16] & $\begin{array}{c}\text { ALFF: right gyrus subcallosum, postcentral gyrus, inferior frontal gyrus left precuneus, superior temporal gyrus, and } \\
\text { middle temporal gyrus }\end{array}$ \\
\hline Liu et al. [17] & ReHo: cognitive, motor, default network, and limbic system \\
\hline
\end{tabular}

TABle 3: Research studies on verum acupuncture vs. sham acupuncture.

\begin{tabular}{lc}
\hline Study & Main findings \\
\hline Zyloney et al. [18] & PAG, PCC, and precuneus; pain matrix and default mode network \\
Zhao et al. [19] & More extensive and remarkable cerebral response \\
Wu et al. [20] & ALFF + ReHo: vision, movement, sensation, emotion, and analgesia \\
Liu et al. [21] & Insula \\
\hline
\end{tabular}

TABLE 4: Research studies contra-acupuncture vs. ipsiacupuncture.

\begin{tabular}{ll}
\hline Study & \multicolumn{1}{c}{ Main findings } \\
\hline Zhang et al. [22] & ReHo: ACC, brainstem-thalamus-cortex \\
Yan et al. [23] & Increased: anterior cingulate and insula \\
& Decreased: anterior/paracingulate cortex \\
\hline
\end{tabular}

connectivity was changed by the xingnaokaiqiao method [29]. Chung et al. [30, 31] pointed out that scalp acupuncture can remarkably enhance the regulation system of the brain network involved in cognition and implementation and the correlation with adjacent brain regions. EA of the auricular concha has an instant effect in modulating the brain default mode network in PI patients, and it may be a brain mechanism underlying improvement of PI [32]. An abdominal acupuncture method could improve the functional connectivities in the cognition network [33]. After electrical stimulation of acupoints, the activities in the frontal lobe, cingulate gyrus, and cerebellum had local changes. In addition, the intensity of changes after 15 minutes were higher than 5 minutes, indicating that the effect of EA on brain functional areas was continuous and strong [34]. Compared with SNA, TENS, TNA, or SNA plus SNA + MS methods showed that the most extensive DMN modulation induced by TNA acupuncture methods can be used as a way of regulating brain activity [35].

\section{Comparison of Different Stimulation Intensities}

Two studies compared the impact of needling at different intensities on brain functional connectivity (Table 6). Shi et al. [36] reported that deep acupuncture with Deqi sensation could regulate neural activity at multiple levels, but no one had Deqi sensation when undergoing shallow acupuncture. Increased connectivity in the MPFC/rACC and dorsolateral prefrontal cortex after enhancement acupuncture compared to standard acupuncture in KOA patients certificated the underlying treatment of the KOA brain mechanism was significantly associated with stimulation intensities [37].

\section{Dominant Diseases}

5.1. Nervous System Diseases. Table 7 shows research on the onset acupuncture mechanism of diagnosis and treatment of dominant diseases. Zheng et al. [38] found that needling LIV3 and LI4 can regulate the functional activity of cognition-related regions in AD patients. Acupuncture at LIV3 and LI4 can increase the hippocampal connectivity in AD disease [39]. Wang et al. [40] demonstrated that acupuncture can achieve antidepressant treatment through modulating limbic system activity, particularly the amygdala and the ACC. The changes of DMN connectivity can be used to monitor CM and acupuncture modulate effects after acupuncture [41]. Stroke patients showed decreased FC in the motor cortex, and the decreased FC was increased after acupuncture [42]. Acupuncture at motor-implicated acupoints specifically modulates the motor-related network in stroke patients and enhances the connectivity between the cerebellum and primary sensorimotor cortex. What's more, acupuncture at motor-implicated acupoints could compensate for the decreased connectivity between the cortex and subcortical areas, thus improving motor coordination and subcortical motor learning ability in stroke patients [43]. Tan et al. [44] indicated that acupuncture at tiaoshenyizhi acupoints can improve cognitive function in mild cognitive impairment disease. Chen et al. [45] demonstrated that ACU at TE5 increased the connectivity of the bilateral sensorimotor networks in ischemic stroke patients. Wang et al. [40] included the action of acupuncture on antidepressant patients may be actualized through regulating the areas of the limbic system. After acupuncture at GB34, the functional connectivity between the PM/SMA and SMG was enhanced in stroke patients, suggesting that acupuncture delays the progression of the disease by increasing the communication connection between the damaged white-matter tracts. [46]. Li et al. [47] observed decreased FC in the RFPN could be reversed by acupuncture. Compared with healthy controls, the connectivity between ACC and PCC was enhanced after acupuncture, and the functions of ACC and PCC were associated with cognitive and motor ability that could be interpreted the modulatory effects of acupuncture [48]. 
TABLe 5: Research studies on different acupuncture methods.

\begin{tabular}{lc}
\hline Methods & Main findings \\
\hline MA + EA, TEAS [26] & MA + EA: more secure and spatially extended connectivity; TEAS: sensorimotor network \\
Laser acupuncture [27] & Cognitive functions \\
TEAS [28] & DMN and SMN \\
XNKQ acupuncture [29] & Motor function \\
Scalp acupuncture [30, 31] & Cognition, implementation network, and adjacent brain regions \\
EA [32] & DMN \\
Abdominal acupuncture [33] & Allomeric function \\
EA [34] & Higher intensity at 15 minutes than 5 minutes \\
SNA, SNA + MS, and TENS [35] & Different acupuncture methods to induce different DMN modulatory effects \\
\hline
\end{tabular}

TABLE 6: Research studies on different stimulation intensities.

\begin{tabular}{lc}
\hline Intensities & Main findings \\
\hline Deep vs. shallow [36] & LPNN and DMN \\
Enhancement vs. standard [37] & MPFC/rACC and dorsolateral prefrontal cortex \\
\hline
\end{tabular}

TABle 7: Research studies on different dominant diseases.

\begin{tabular}{|c|c|}
\hline Dominant diseases & Main findings \\
\hline $\mathrm{AD}[38]$ & Cognition regions \\
\hline $\mathrm{AD}[39]$ & Hippocampal \\
\hline Depression $[40]$ & Limbic system, amygdala, and the ACC \\
\hline $\mathrm{CM}[41]$ & $\mathrm{DMN}$ \\
\hline Stroke $[42]$ & Bilateral motor cortex \\
\hline Stroke $[43]$ & Motor-related network \\
\hline MCI [44] & Cognition regions \\
\hline Ischemic stroke [45] & Sensorimotor network \\
\hline Depression $[40]$ & Limbic system, amygdala, and ACC \\
\hline Stroke $[46]$ & PM/SMA and SMG RFPN \\
\hline Migraine [47] & ACC and PCC \\
\hline Stroke [48] chronic pain $[49-51]$ & Networks: DMN, salience, central executive, and sensorimotor \\
\hline ALBP $[52]$ & Limbic, pain, attentional and somatosensory system, and DMN \\
\hline $\mathrm{CD}[53]$ & Afferent processing network and DMN \\
\hline Smoking craving [54] & $\begin{array}{lll}\mathrm{S} N \\
\mathrm{H}\end{array}$ \\
\hline Hypertension [55] & Frontal lobe, cerebellum, and insula \\
\hline PMS [56] & Aberrant neural activity \\
\hline Cardiovascular [57] & Cortical, hypothalamus and brainstem \\
\hline
\end{tabular}

5.2. Motor System Diseases. Imaging studies evidenced that acupuncture may achieve its therapeutic effect through regulating the connectivity of DMN, salience, central executive, and sensorimotor networks in chronic pain patients [49-51]. Wu et al. [52] showed that acupuncture causes extensive inactivation of almost all limbic systems, pain systems, and DMN in patients with acute lower back pain, which indicated that multiplex networks involved in the treatment of motor system diseases by acupuncture.

5.3. Other Diseases. Bao et al. [53] found that the efficacy of acupuncture on $\mathrm{CD}$ may involve the regulation of the afferent processing network and DMN in the brain. The effects of acupuncture in treating smoking craving were remarkable, and the SN played an important role in the treatment course [54]. The efficacy of LR3 in connectivity was more concentrated in the frontal lobe, cerebellum, and insula [55]. Pang et al. [56] found that the aberrant neural activity of PMS patients could be regulated by acupuncture at SP6. Acupuncture may adjust the cardiovascular system through multiple brain networks with the cortical level, the hypothalamus, and the brainstem [57].

\section{Conclusions}

This review demonstrates the application of rs-fMRI in the study of the acupuncture mechanism from the aspects of study acupoints, subjects, acupuncture methods, and intensities and found that the application of rs-fMRI in acupuncture research mainly includes research on the onset mechanism of acupuncture treatment; visual evidence of diagnosis and treatment of dominant diseases; efficacy assessments; physiological mechanism of acupoints 
stimulation; and specific visualization of acupoints. Specifically, the following conclusions can be drawn from the physiological mechanism of acupuncture at acupoints and its specificity research: there are differences in the brain connectivity and local activities between single and different acupoints, and combination points have more wide activate areas than single point; the regulate areas are mostly related to the emotional, cognitive, and painful functions; limbic system and subcortical areas are found to be hubs after acupuncture; verum acupuncture may increase DMN, PAG, PCC, and pain matrix connectivity compared with sham acupuncture, and sham acupuncture influenced less functional areas than true acupuncture; the local brain functional activities of ipsilateral acupuncture are different from those of contralateral acupuncture; the effect of acupuncture has an obvious individual difference; and there are different degrees of changes in brain functional connectivity among different acupunctures, intensities, methods, and different subjects, and an adjusting acupuncture approach can be used as a means of regulating brain activity. In addition, in the study of the onset mechanism of acupuncture treatment, diagnosis and treatment of dominant diseases, and curative effect evaluation, we can see that the brain functional connectivity is not the same in several dominant diseases, acupuncture can treat diseases by regulating the brain network related to cognitive and motor functions, rs-fMRI has an important meaning for the evaluation of the therapeutic effect after acupuncture, and there is a great significance for the establishment of disease-specific biomarkers.

A good technical platform has been offered by the development of medical imaging for the study of acupuncture mechanisms. With the help of fMRI, the inherent and spontaneous neural activities of neurons can be observed from the microscale, mesoscale, and the entire brain area, and it will contribute to depict the organization and mechanism of the brain. However, the sample size is relatively small. In the future, we will collect more samples to expand the database for developing an artificial intelligence algorithm and automatic result output system of fMRI images to explore the cerebral mechanism of acupuncture.

\section{Abbreviations}

STG: $\quad$ Superior temporal gyrus

ReHo: Regional homogeneity

ALFF: Low-frequency fluctuations

PAG: Periaqueductal gray

PCC: Posterior cingulate cortex

ACC: Anterior cingulate cortex

MA: Manual acupuncture

EA: Electroacupuncture

TEAS: Transcutaneous electrical acupoint stimulation

TGA: Thirteen ghost acupoints

MTEAS: Minimal TEAS

PI: $\quad$ Primary insomnia

DMN: Default mode network

SNA: Single-needle acupuncture

TENS: Transcutaneous electrical nerve stimulation
TNA: Three-needle acupuncture

LPNN: Limbic-paralimbic-neocortical network

MPFC: Medial prefrontal cortex

rACC: Rostral anterior cingulate cortex

KOA: Knee osteoarthritis

CM: $\quad$ Chronic migraine

SMA: Supplementary motor area

SMG: Supramarginal gyrus

RFPN: Right frontoparietal network

PMS: Premenstrual syndrome

\section{Conflicts of Interest}

The authors have no conflicts of interest to disclose.

\section{Authors' Contributions}

Xiaoling Li, Lina Cai, and Xiaoxu Jiang contributed equally to this work.

\section{Acknowledgments}

This work was supported in part by the National Foundation of Natural Science of China (Nos. 81973930, 82074537, 81373714, and 82074539), Natural Science of Heilongjiang Province (Nos. LH2020H103 and H2016081), and the Fund of Heilongjiang Province Traditional Medicine Research (No. ZHY12-Z046)

\section{References}

[1] Z. Yu, Y. W. Yu, Y. L. Yu et al., "Imaging OF brain function based on the analysis of functional connectivity-imaging analysis of brain function by FMRI after acupuncture," $A f$ rican Journal of Traditional Complementary \& Alternative Medicines, vol. 13, no. 6, pp. 90-100, 2016.

[2] S. M. Smith, "The future of FMRI connectivity," Neuroimage, vol. 62, no. 2, pp. 1257-1266, 2012, [.

[3] H. Yu, X. Wu, L. Cai, B. Deng, and J. Wang, "Modulation of spectral power and functional connectivity in human brain by acupuncture stimulation," IEEE Transactions on Neural Systems and Rehabilitation Engineering, vol. 26, no. 5, pp. 977986, 2018.

[4] B. E. Scheffold, C. L. Hsieh, and G. Litscher, "Neuroimaging and neuromonitoring effects of electro and manual acupuncture on the central nervous system: aliterature review and analysis," Evidence-Based Complementary and Alternative Medicine, vol. 2015, Article ID 641742, 29 pages, 2015.

[5] R. Sun, Y. Yang, Z. Li et al., "Connectomics: a new direction in research to understand the mechanism of acupuncture," Evidence-Based Complementary and Alternative Medicine, vol. 2014, Article ID 568429, 9 pages, 2014.

[6] R. L. Buckner, F. M. Krienen, and B. T. T. Yeo, "Opportunities and limitations of intrinsic functional connectivity MRI," Nature Neuroscience, vol. 16, no. 7, pp. 832-837, 2013.

[7] C. Zhong, L. Bai, R. Dai et al., "Modulatory effects of acupuncture on resting-state networks: a functional MRI study combining independent component analysis and multivariate granger causality analysis," Journal of Magnetic Resonance Imaging, vol. 35, no. 3, pp. 572-581, 2012.

[8] W. Qiu, B. Yan, H. He et al., "Dynamic functional connectivity analysis of taichong (LR3) acupuncture effects in various 
brain regions," Neural Regeneration Research, vol. 7, no. 6, pp. 451-456, 2012.

[9] Y. Feng, L. Bai, Y. Ren et al., "Investigation of the large-scale functional brain networks modulated by acupuncture," Magnetic Resonance Imaging, vol. 29, no. 7, pp. 958-965, 2011.

[10] X. Long, W. Huang, V. Napadow et al., "Sustained effects of acupuncture stimulation investigated with centrality mapping analysis," Frontiers in Human Neuroscience, vol. 10, p. 510, 2016.

[11] Y. Zheng, Y. Wang, Y. Lan et al., "Imaging of brain function based on the analysis of functional connectivity-imaging analysis of brain function by FMRI after acupuncture at LR3 IN healthy individuals," African Journal of Traditional, Complementary and Alternative Medicines, vol. 29, no. 13, pp. 90-100, 2016.

[12] R. Cai, Y. Guan, H. Wu et al., "Effects on the regional homogeneity of resting-state brain function in the healthy subjects of gastric distention treated with acupuncture at the front-mu and back-shu points of the stomach, Weishu (BL 21) and Zhongwan (CV 12)," Zhongguo Zhen Jiu, vol. 12, no. 38, pp. 379-386, 2018.

[13] T. Nierhaus, D. Pach, W. Huang et al., "Differential cerebral response to somatosensory stimulation of an acupuncture point vs.two non-acupuncture points measured with EEG and fMRI," Frontiers in Human Neuroscience, vol. 9, p. 74, 2015.

[14] L. Liu, Y. Wu, J. Zheng et al., "Cerebral activation effects of acupuncture using zusanli (ST36) and yanglingquan (GB34) points based on regional homogeneity indices: a resting-state fMRI study," Journal of X-Ray Science and Technology, vol. 24, no. 2, pp. 297-308, 2016.

[15] S. Q. Zhang, C. Z. Tang, Y. J. Wang et al., "Brain activation and inhibition after acupuncture at taichong and taixi: restingstate functional magnetic resonance imaging," Neural Regeneration Research, vol. 10, no. 2, pp. 292-297, 2015.

[16] Y. L. Zhou, C. G. Su, S. F. Liu et al., "Amplitude changes of low frequency fluctuation in brain spontaneous nervous activities induced by needling at hand taiyin Lung channel," Zhongguo Zhong Xi Yi Jie He Za Zhi, vol. 36, no. 5, pp. 553-558, 2016.

[17] L. Liu, S. Chen, D. Zeng, H. Li, C. Shi, and L. Zhang, "Cerebral activation effects of acupuncture at Yanglinquan(GB34) point acquired using resting-state fMRI," Computerized Medical Imaging and Graphics, vol. 67, pp. 55-58, 2018.

[18] C. E. Zyloney, K. Jensen, G. Polich et al., "Imaging the functional connectivity of the periaqueductal gray during genuine and sham electroacupuncture treatment," Molecular Pain, vol. 6, p. 80, 2010.

[19] L. Zhao, J. Liu, F. Zhang et al., "Effects of long-term acupuncture treatment on resting-state brain activity in migraine patients: a randomized controlled trial on active acupoints and inactive acupoints," European Journal of Integrative Medicine, vol. 6, no. 6, p. 703, 2014.

[20] C. Wu, S. Qu, J. Zhang et al., "Correlation between the effects of acupuncture at taichong (LR3) and functional brain areas: a resting-state functional magnetic resonance imaging study using true versus sham acupuncture," Evidence-Based Complementary and Alternative Medicine, vol. 2014, Article ID 729091, 7 pages, 2014.

[21] J. Liu, W. Qin, Q. Guo et al., "Divergent neural processes specific to the acute and sustained phases of verum and SHAM acupuncture," Journal of Magnetic Resonance Imaging, vol. 33, no. 1, pp. 33-40, 2010.

[22] S. Zhang, X. Wang, C.-Q. Yan et al., "Different mechanisms of contralateral-or ipsilateral-acupuncture to modulate the brain activity in patients with unilateral chronic shoulder pain: a pilot fMRI study," Journal of Pain Research, vol. 11, no. 11, pp. 505-514, 2018.

[23] C.-Q. Hu, J.-W. Huo, Xu Wang et al., "Different degree centrality changes in the brain after acupuncture on contralateral or ipsilateral acupoint in patients with chronic shoulder pain: a resting-state fMRI study," Neural Plasticity, vol. 2020, Article ID 5701042, 11 pages, 2020.

[24] X. Yang, J. Gong, L. Jin, L. Liu, J. Sun, and W. Qin, "Effect of catechol-O-methyltransferase Val158Met polymorphism on resting-state brain default mode network after acupuncture stimulation," Brain Imaging and Behavior, vol. 12, no. 3, pp. 798-805, 2018.

[25] La-M. Li, L. ü Fa-Jin, Z.-J. Guo et al., "Effect of acupuncture stimulation of zusanli (ST 36) on cerebral regional homogeneity in volunteer subjects with different constitutions: a resting-state fMRI study," Zhen Ci Yan Jiu, vol. 38, no. 4, pp. 306-313, 2013.

[26] Y. Jiang, H. Wang, Z. Liu et al., "Manipulation of and sustained effects on the human brain induced by different modalities of acupuncture: an fMRI study," PLoS One, vol. 8, no. 6, Article ID e66815, 2013.

[27] J. Lv, C. Shi, Y. Deng et al., "The brain effects of laser acupuncture at thirteen ghost acupoints in healthy individuals: a resting-state functional MRI investigation," Computerized Medical Imaging and Graphics, vol. 54, pp. 48-54, 2016.

[28] Y. Jiang, Y. Hao, Y. Zhang et al., "Thirty minute transcutaneous electric acupoint stimulation modulates resting state brain activities: a perfusion and bold fMRI study," Brain Research, vol. 1457, pp. 13-25, 2012.

[29] T. Nierhaus, Y. Chang, B. Liu et al., "Somatosensory stimulation with XNKQ acupuncture modulates functional connectivity of motor areas," Frontiers in Neuroscience, vol. 11, no. 13, p. 147, 2019.

[30] W.-Y. Chung, S.-Y. Liu, J.-C. Gao et al., "Modulatory effect of international standard scalp acupuncture on brain activation in the elderly as revealed by resting-state fMRI," Neural Regeneration Research, vol. 14, no. 12, pp. 2126-2131, 2019.

[31] J. Cao, Y. Huang, N. Meshberg, S. A. Hodges, and J. Kong, "Neuroimaging-Based scalp acupuncture locations for dementia," Journal of Clinical Medicine, vol. 9, no. 8, p. 2477, 2020.

[32] B. Zhao, Li Liang, J.-L. Zhang et al., "Instant adjustive effect of auricular electroacupuncture on brain default model network of patients with primary insomnia," Zhen Ci Yan Jiu, vol. 25, no. 12, pp. 884-887, 2019.

[33] Z.-P. Zhong, S.-S. Wu, Z.-G. Chen et al., "Study on response of resting-state functional magnetic resonance imaging induced by abdominal acupuncture with invigorating the kidney and nourishing marrow method," Zhongguo Zhen Jiu, vol. 31, no. 2, pp. 139-143, 2011.

[34] Y. Zheng, S. Qu, N. Wang et al., "Post-stimulation effect of electroacupuncture at yintang (EX-HN3) and GV20 on cerebral functional regions in healthy volunteers: a resting functional MRI study," Acupuncture in Medicine, vol. 30, no. 4, pp. 307-315, 2012.

[35] Y.-J. Lin, Y.-Y. Kung, W.-J. Kuo et al., "Effect of acupuncture "dose"-on modulation of the default mode network of the brain," Acupuncture in Medicine, vol. 34, no. 6, pp. 425-432, 2016.

[36] Y. Shi, S. Zhang, Q. Li et al., "A study of the brain functional network of deqi via acupuncturing stimulation at BL40 by rsfMRI," Complementary Therapies in Medicine, vol. 25, pp. 71-77, 2016. 
[37] J. Kong, Z. Wang, J. Leiser et al., "Enhancing treatment of osteoarthritis knee pain by boosting expectancy: a functional neuroimaging study," NeuroImage: Clinical, vol. 18, pp. 325-334, 2018.

[38] W. Zheng, Z. Su, X. Liu et al., "Modulation of functional activity and connectivity by acupuncture in patients with Alzheimer disease as measured by resting-state fMRI," PLoS One, vol. 13, no. 5, Article ID e0196933, 2018.

[39] Z. Wang, P. Liang, Z. Zhao et al., "Acupuncture modulates resting state hippocampal functional connectivity in alzheimer disease," PLos One, vol. 9, no. 3, Article ID e91160, 2014.

[40] X. Wang, Z. Wang, J. Liu et al., "Repeated acupuncture treatments modulate amygdala resting state functional connectivity of depressive patients," NeuroImage: Clinical, vol. 12, no. C, pp. 746-752, 2016.

[41] Y. Zou, W. Tang, X. Li, M. Xu, and J. Li, “Acupuncture reversible effects on altered default mode network of chronic migraine accompanied with clinical symptom relief," Neural Plasticity, vol. 2019, Article ID 5047463, 10 pages, 2019.

[42] Y. Ning, K. Li, C. Fu et al., "Enhanced functional connectivity between the bilateral primary motor cortices after acupuncture at yanglingquan (GB34) in right-hemispheric subcortical stroke patients: a resting-state fMRI study," Frontiers in Human Neuroscience, vol. 10, no. 11, p. 178, 2017.

[43] Z. Xie, F. Cui, Y. Zou et al., "Acupuncture enhances effective connectivity between cerebellum and primary sensorimotor cortex in patients with stable recovery stroke," Evidence-Based Complementary and Alternative Medicine, vol. 2014, no. 3, 9 pages, Article ID 603909, 2014.

[44] T. T. Tan, D. Wang, J. K. Huang et al., "Modulatory effects of acupuncture on brain networks in mild cognitive impairment patients," Neural Regeneration Research, vol. 12, no. 2, pp. 250-258, 2017.

[45] J. Chen, J. Wang, Y. Huang et al., "Modulatory effect of acupuncture at waiguan (TE5) on the functional connectivity of the central nervous system of patients with ischemic stroke in the left basal ganglia," PLoS One, vol. 9, no. 6, Article ID e96777, 2014.

[46] X. Han, B. LijunC. Sun et al., Acupuncture enhances communication between cortices with damaged white matters in poststroke motor impairment," Evidence-Based Complementary and Alternative Medicine, vol. 2019, Article ID 4245753, 11 pages, 2019.

[47] K. Li Zhang, Y. Zhang, Y. Ning et al., "The effects of acupuncture treatment on the right frontoparietal network in migraine without aura patients," The Journal of Headache Pain, vol. 16, p. 518, 2015.

[48] Y. Zhang, K. Li, Yi Ren et al., "Acupuncture modulates the functional connectivity of the default mode network in stroke patients," Evidence-Based Complementary and Alternative Medicine, vol. 2014, Article ID 765413, 7 pages, 2014.

[49] N. Egorova, R. L. Gollub, and J. Kong, "Repeated verum but not placebo acupuncture normalizes connectivity in brain regions dysregulated in chronic pain," NeuroImage: Clinical, vol. 9, pp. 430-435, 2015.

[50] Y. Tu, A. Ortiz, R. L. Gollub et al., "Multivariate resting-state functional connectivity predicts responses to real and sham acupuncture treatment in chronic low back pain," NeuroImage: Clinical, vol. 23, Article ID 101885, 2019.

[51] X. Chen, B. Rosa, S. G. Freeman et al., "The modulation effect of longitudinal acupuncture on resting state functional connectivity in knee osteoarthritis patients," Molecular Pain, vol. 29, no. 11, p. 67, 2015.
[52] Yu Shi, Z. Liu, S. Zhang et al., "Brain network response to acupuncture stimuli in experimental acute low back pain: an fMRI study," Evidence-Based Complementary and Alternative Medicine, vol. 2015, Article ID 210120, 13 pages, 2015.

[53] C. Bao, D. Wang, P. Liu et al., "Effect of electro-acupuncture and moxibustion on brain connectivity in patients with crohn's disease: a resting-state fMRI study," Frontiers in Human Neuroscience, vol. 11, p. 559, 2017.

[54] Y.-Y. Wang, Z. Liu, F. Chen et al., "Effects of acupuncture on craving after tobacco cessation: a resting-state fMRI study based on the fractional amplitude of low-frequency fluctuation," Quantitative Imaging in Medicine and Surgery, vol. 9, no. 6, pp. 1118-1125, 2019.

[55] Yu Zheng, J. Zhang, Y. Wang et al., “Acupuncture decreases blood pressure related to hypothalamus functional connectivity with frontal lobe, cerebellum, and insula: a study of instantaneous and short-term acupuncture treatment in essential hypertension," Evidence-Based Complementary and Alternative Medicine, vol. 2016, Article ID 6908710, 10 pages, 2016.

[56] Y. Pang, H. Liu, G. Duan et al., “Altered brain regional homogeneity following electro-acupuncture stimulation at sanyinjiao (SP6) in women with premenstrual syndrome," Frontiers in Human Neuroscience, vol. 31, no. 12, p. 104, 2018.

[57] H. Chen, J. Dai, X. Zhang et al., "Hypothalamus-related resting brain network underlying short-term acupuncture treatment in primary hypertension," Evidence-Based Complementary and Alternative Medicine, vol. 2013, Article ID 808971, 9 pages, 2013. 